\title{
PtBi Alloy Nanoparticles on Reduced Graphitic Oxide Support for Electrocatalysis
}

\author{
Shalini Tripathi ${ }^{1}$ and N Ravishankar ${ }^{1}$ \\ 1. Materials Research Center, Indian Institute of Science, Bangalore, India.
}

Pt nanoparticles on suitable supports are commonly employed as electrocatalysts to improve the slow kinetics of methanol oxidation [1]. However, it is not cost-effective and suffers from two serious limitations. Firstly, in the course of methanol oxidation, the evolution of $\mathrm{CO}$ gas poisons the Pt-catalyst leading to its reduced cyclic stability and secondly, Pt nanoparticles (NPs) undergo coarsening at the operative voltage in fuel cell, gradually losing its activity. In this context, alloying Pt with a non-noble element along with achieving a dispersion of the catalyst on a conducting support can solve these problems. We have demonstrated earlier that dispersion of ultrafine Pt NPs on a reduced graphitic oxide (rGO) is achievable through a microwave (MW) assisted synthesis route [2], as shown in Figure 1 (a, e,f). However, achieving an alloy/intermetallic nanoparticle catalyst still remains a challenge owing to the difficulties associated with the co-reduction of two metals with widely different reduction potentials. In this work, we report a MW-based wet chemical approach for alloying Pt NPs with Bi on rGO support that enhances the stability of the catalyst. In the MW-assisted ultrafast route, we were able to synthesize $\mathrm{Pt}-\mathrm{Bi}$ intermetallic nanoparticles on rGO support upon reaction. X-ray diffraction (XRD) shows the formation of PtBi alloy phases with two distinct intermetallic compositions (Figure 1 (g)), hexagonal PtBi phase, along with rhombohedral $\mathrm{PtBi}_{2}$ phase. Figure 1 (c) shows high resolution transmission electron (HRTEM) image of PtBi intermetallic nanoparticle showing fringes corresponding to (100) planes of the hexagonal structure. Scherrer analysis from the XRD pattern and detailed scanning transmission electron microscopy (STEM) coupled with EDS of the particles reveal that the larger particles have $\mathrm{PtBi}_{2}$ stoichiometry, and the smaller ones have a PtBi stoichiometry (Figure 2).

Owing to the MW-assisted reaction, the NPs are capping-free as well and there is no detectable amount of oxide phases formed. The calculated phase diagram of Pt-Bi system shows three line compounds, $\mathrm{PtBi}_{2}, \mathrm{Bi}_{3} \mathrm{Pt}_{2}$ and $\mathrm{PtBi}$. $\mathrm{Bi}_{3} \mathrm{Pt}_{2}$ is a high temperature phase and hence its absence in our reaction product is not surprising. In spite of a difference in reduction potential of $\mathrm{Pt}^{4+} / \mathrm{Pt}^{0}$ and $\mathrm{Bi}^{3+} / \mathrm{Bi}^{0}$, the use of relatively high temperature in the microwave synthesis leads to the reduction of both $\mathrm{Pt}$ and $\mathrm{Bi}$ precursors.

Having a higher electronegativity, $\mathrm{Bi}$ is expected to deplete the electron density of Pt atoms near Fermi energy compared to that in bare Pt. In the methanol oxidation, the alloy catalyst exhibits an increase in current upon cycling, probably because of Bi leaching from the catalyst. However, upon cycling, the methanol oxidation current for the alloy catalyst is higher compared to that of Pt-based catalyst, depicting the higher stability. Moreover, chronoamperometric study reinforces the point of stability of the alloy catalyst, as it achieves a higher current upon cycling, unlike that of pristine Pt-based catalyst, where the current is found to decay because of poisoning [3].

\section{References:}

[1] Zhao, X. et al., Energy Envir. Sci., 4, (2011), 2736-2753.

[2] Kundu, P. et al., Chem. Mater., 23, (2011), 2772-2780.

[3] The authors acknowledge funding from the Department of Science and Technology (DST), India and also the TEM facilities provided by the Advanced Facility for Microscopy and Microanalysis (AFMM), Indian Institute of Science, Bangalore, India. 




Figure 1. Low-magnification TEM images showing the morphology of nucleated (a) Pt and (b) PtBi NPs on the rGO sheets; (c) HRTEM images showing $\{100\}$ and $\{102\}$ lattice spacings of intermetallic $\mathrm{PtBi}$ and (d) $\mathrm{PtBi}_{2}$ phases respectively; (e) SAED pattern and (f) HRTEM image of nucleated Pt on rGO support showing the presence of crystalline Pt NPs; $(\mathrm{g})$ XRD pattern depicting the formation of mixed intermetallic phases $\left(\mathrm{PtBi}\right.$ and $\left.\mathrm{PtBi}_{2}\right)$.
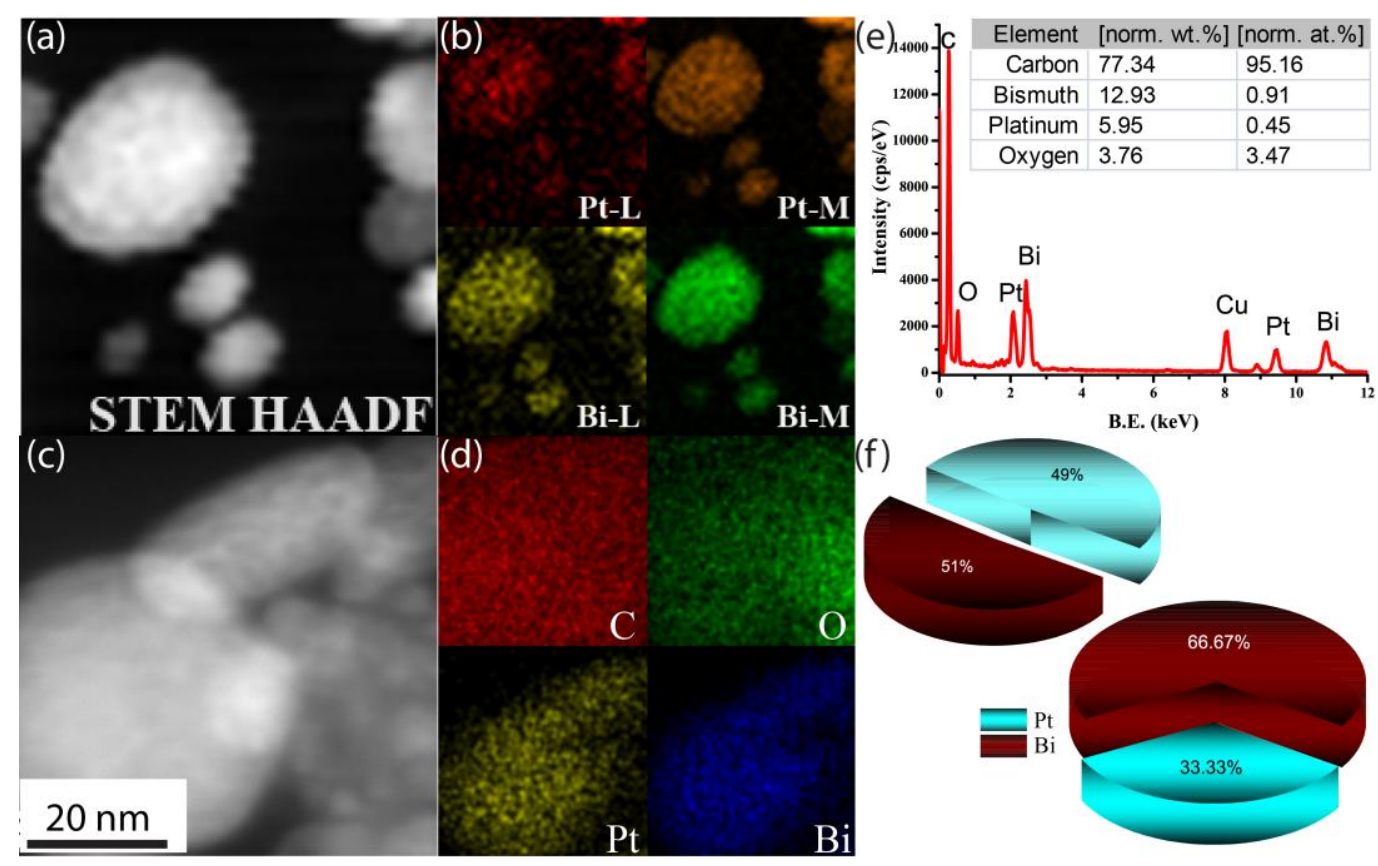

Figure 2. (a) STEM-HAADF image of the 5-20 nm nanoparticles on rGO sheet; (b) STEM-EDS elemental mapping shows homogenous elemental distribution of $\mathrm{Pt}$ and $\mathrm{Bi}$ in the particles; (c) STEMHAADF image of the larger nanoparticle (>20 nm) on rGO sheet; (d) Elemental mapping; (e) EDS spectrum \& quantification results and (f) elemental composition of the NPs shown in (a) and (c) respectively, clearly showing that the smaller particles are $\mathrm{PtBi}$, while the larger ones are $\mathrm{PtBi}_{2}$. 